\title{
Mycobacterium tuberculosis detection from oral swabs with Xpert MTB/RIF ULTRA: a pilot study
}

\author{
Annelies W. Mesman ${ }^{1 *}\left(\mathbb{D}\right.$, Roger Calderon², Martin Soto ${ }^{2}$, Julia Coit ${ }^{1}$, Juan Aliaga², Milagros Mendoza ${ }^{2}$ \\ and Molly F. Franke ${ }^{1}$
}

\begin{abstract}
Objective: Diagnostic testing for tuberculosis depends on microbiological detection of Mycobacterium tuberculosis (Mtb) in sputum. For patients unable to expectorate sputum, such as children and individuals living with HIV, this poses barriers to rapid diagnosis and treatment initiation. Therefore, this study aimed to use oral swabs as an alternative sample type for Mtb detection via molecular testing.

Results: In a pilot study, we aimed to evaluate sensitivity of Mtb detection via oral swabs using Xpert MTB/RIF ULTRA. We enrolled 33 TB cases and 30 controls from Lima, Peru, and detected Mtb from oral swabs with a sensitivity of 45\% (95\% confidence interval (CI) 29-62\%) and specificity of 100\% (95\% Cl 89-100\%) using liquid culture of sputum as reference test. Our current protocol will need optimization, but these results support future exploration of the use of oral swabs for Mtb detection.
\end{abstract}

Keywords: Molecular diagnostics, Sample type, Buccal swab, Xpert

\section{Introduction}

Timely tuberculosis (TB) diagnosis and treatment initiation is hampered in patients unable to produce a sputum sample for microbiological confirmation of Mycobacterium tuberculosis $(M t b)$ (e.g., children and individuals living with HIV). Recent research efforts have aimed to identify alternative, non-invasive sample types for molecular detection of TB disease in these patient groups.

The results of two recent studies $[1,2]$ show successful molecular detection of $M t b$ from oral swabs, after collection in Tris-EDTA-Sucrose-SDS lysis buffer, followed by DNA extraction and IS6110 qPCR. Oral swabs would be an ideal alternative sample type to sputum-collection is easy with a minimal risk on aerosol production. However, DNA extraction and in-house developed amplification assays are laborious procedures that require bio-safety level laboratory facilities. The rollout of Xpert MTB/RIF

\footnotetext{
*Correspondence: annelies_mesman@hms.harvard.edu

${ }^{1}$ Department of Global Health and Social Medicine, Harvard Medical School, Boston, USA

Full list of author information is available at the end of the article
}

(Cepheid Sunnyvale, USA) and its subsequent recommended use by WHO as an initial diagnostic tool in 2013, introduced automated molecular testing to many laboratories in resource-limited settings. Recently, Cepheid introduced the novel MTB/RIF Ultra assay. This test is more sensitive than MTB/RIF, albeit at the cost of a slight reduction in specificity [3], and has improved $M t b$ detection in smear-negative respiratory samples [3, 4], and therefore could be a valuable test for oral swab testing.

\section{Main text \\ Study aim}

In a small proof-of-concept pilot study we estimated the sensitivity and specificity of $M t b$ detection from oral swabs using Xpert MTB/RIF ULTRA (Cepheid, Sunnyvale, USA). A second aim of this pilot was to pilot three collection methods and storage procedures of swabs. In addition to the method described previously [1,2], which requires preparation of a buffer, we employed two other methods that could facilitate implementation of Xpert testing of oral swabs in low-resource settings.

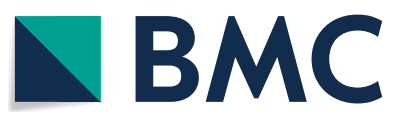

C The Author(s) 2019. This article is distributed under the terms of the Creative Commons Attribution 4.0 International License (http://creativecommons.org/licenses/by/4.0/), which permits unrestricted use, distribution, and reproduction in any medium, provided you give appropriate credit to the original author(s) and the source, provide a link to the Creative Commons license, and indicate if changes were made. The Creative Commons Public Domain Dedication waiver (http://creativecommons.org/ publicdomain/zero/1.0/) applies to the data made available in this article, unless otherwise stated. 


\section{Methods}

We recruited 33 adults diagnosed with pulmonary TB from TB clinics in Lima, Peru prior to anti-TB treatment initiation. Thirty controls (i.e., clinic attendees with no respiratory symptoms) were recruited from the same health centers. Participants were randomly assigned to one of three sample collection groups to assess collection methods and storage procedures: (1) Omniswab (Whatman, catalog \#WB100035) in lysis buffer as described in $[1,2]$; (2) Omniswab in phosphate buffered saline (PBS); (3) Sample capture on FTA cards (Whatman catalog \#120237). We collected a single oral cheek swab from all TB patients and controls, and a sputum sample from TB patients. Sputum was used for microbiological confirmation via microscopy following Ziehl-Neelsen staining and liquid culture (i.e., the gold reference standard; in BACTEC MGIT 960, BD Franklin Lakes, USA). For Xpert testing, 500-600 $\mu \mathrm{l}$ of sample in PBS or lysis buffer was mixed in a 1:2 ratio with Xpert sample reagent buffer; sample reagent was directly added to FTA cards. Manufacturer's guidelines were followed for all remaining steps of testing. We calculated sensitivity and specificity, and corresponding exact $95 \%$ confidence intervals (CI), via Episheet (http://www.krothman.org/episheet.xls).

\section{Results}

All 33 TB patients had a positive sputum liquid culture result. The sputum of four patients tested negative in smear microscopy. The overall sensitivity for oral swab detection was $45 \%$ (95\% confidence interval (CI) 29-62\%), with positive tests for $15 / 33$ TB patients (Table 1a). Specificity was 100\% (95\% CI 89-100\%), (26/26 of controls tested negative; 4 tests had invalid results due to power interruption). We observed a higher sensitivity for patients with a smear-positive compared to a smear negative sputum sample, although we included too few patients for statistical comparison (Table 1b). Sensitivity was similar across the three swab collection protocols, with swab collection in Tris-EDTA buffer resulting in $M t b$ detection in 6 of 10 patients, followed by FTA and PBS cards (5/13 and 4/10 respectively; Table 1b).

Three of the 33 patients were diagnosed with drug resistant TB based on culture-based drug sensitivity testing. We confirmed rifampin resistance with Xpert oral swab analysis for one of these patients, $M t b$ was not detected in the swabs of the other two patients.

\section{Discussion}

These results support the possible utility of oral swab samples for TB detection. Luabeya et al. reported that Omniswab tongue swabs yielded significant better results compared to cheek swabs, with a sensitivity of $71.2 \%$ for a single swab and $83.1 \%$ for two swabs in 59 patients (case definition based on MTB/RIF Xpert or liquid culture) and $83.1 \%$ for single swab in another group of 89 patients (compared to Xpert MTB/RIF) [2]. Their additional use of sputum Xpert as the reference test and tongue swab may in part explain the higher observed sensitivity in their study and would be interesting adjustments to the Xpert protocol. Their overall specificity was $91.5 \%$, which would be below acceptable standards for a novel TB test, as described in the WHO target product profile [5]. The authors hypothesize that the limited specificity was due to contamination errors in the PCR step. The automated integrated Xpert system is less prone to contamination, and the $100 \%$ specificity we measured in this pilot study is promising. Additionally, although current numbers are too small to draw any conclusions, these preliminary results suggest that other collection and storage procedures can be applied for oral swab testing, but they may be inferior to Omniswab. In conclusion, these data

Table 1 (a) Overall diagnostic assay performance, (b) Xpert positivity per collection method, stratified by smear microscopy result of the TB cases

\begin{tabular}{|c|c|c|c|c|c|c|}
\hline & & \multicolumn{3}{|c|}{ Mtb detection $\mathrm{n} / \mathrm{N}$} & \multirow{2}{*}{\multicolumn{2}{|c|}{ Sensitivity $(95 \% \mathrm{Cl})$ Specificity $(95 \% \mathrm{Cl}$}} \\
\hline & & TB cases & & Controls & & \\
\hline \multicolumn{7}{|l|}{ (a) } \\
\hline & & $15 / 33$ & & $0 / 26$ & $45 \%(29-62)$ & $100 \%(89-100)$ \\
\hline Subgroup & $\begin{array}{l}\text { Lysis buffer } \\
\text { n/N }\end{array}$ & $\begin{array}{l}\text { FTA } \\
\mathrm{n} / \mathrm{N}\end{array}$ & $\begin{array}{l}\text { PBS } \\
\text { n/N }\end{array}$ & $\begin{array}{l}\text { Combined } \\
\mathrm{n} / \mathrm{N}\end{array}$ & Sensitivity $(95 \% \mathrm{Cl})$ & Specificity $(95 \% \mathrm{Cl})$ \\
\hline \multicolumn{7}{|l|}{ (b) } \\
\hline SM positive cases & $6 / 10$ & $4 / 10$ & $4 / 9$ & $14 / 29$ & $48 \%(31-66)$ & \\
\hline SM negative cases & $0 / 0$ & $1 / 3$ & $0 / 1$ & $1 / 4$ & $25 \%(1.2-76)$ & \\
\hline Controls & $0 / 7$ & $0 / 10$ & $0 / 9$ & $0 / 26$ & & $100 \%(89-100)$ \\
\hline
\end{tabular}

SM smear 
support the feasibility of $M t b$ detection in oral swabs using Xpert. Future work could explore the utility of oral swabs for the diagnosis of TB in patients unable to produce sputum and optimization of collection and storage strategies.

\section{Limitations}

The major limitation of this study is the small sample size and pooling results of samples obtained by different collection methods. These numbers are insufficient to test for differences in sensitivity across the collection methods. A second limitation is that we enrolled asymptomatic controls, whereas symptomatic individuals will be the most important group in which to evaluate the specificity of this test. Because of our case-control design, we could not directly calculate the positive and negative predictive values; these values would be an artefact of the study design and not generalizable. We hope to calculate predictive values in a future study, in which symptomatic patients are consecutively enrolled and the number of cases reflects disease prevalence in the study population. Finally, since we tested swabs from adult patients with culture-confirmed TB, this could overestimate the utility of this test for patients that would benefit most from oral swab testing (i.e., those with paucibacillary disease or who are unable to produce a sputum sample).

\section{Abbreviations}

Cl: confidence interval; MTB: Mycobacterium tuberculosis; PBS: phosphate buffered saline; SM: smear; TB: tuberculosis; RIF: rifampin; WHO: World Health Organization.

\section{Acknowledgements}

We are grateful for all participants in this study. We thank Socios En Salud for their contribution and dedication to health equity.

\section{Authors' contributions}

AM: Study design, led the data analysis and interpretation and wrote the manuscript. RC: study design and data analysis. MS: study implementation and interpretation of results. JC: study implementation and interpretation of results JA: study implementation. MM: study implementation. MF: conceptualized and designed the study and contributed to writing and editing. All authors critically reviewed the manuscript. All authors read and approved the final manuscript.

\section{Funding}

This work was funded by the National Institutes of Health $(\mathrm{NIH})$ under the Center of Excellence in Translational Research (CETR) grant U19 Al109755. The funders had no role in the study design, data collection and analysis, decision to publish, or preparation of the manuscript.

\section{Availability of data and materials}

Reported data are available on request from the corresponding author.

\section{Ethics approval and consent to participate}

Study participants provided written informed consent to participate. This pilot was part of a study approved by Ethics Committee of Peru's National Institute of Health and the Office of Human Research Administration at the Harvard Medical School.

\section{Consent to publish}

Consent to publish is not applicable, we do not report individual patient data.

\section{Competing interests}

The authors declare that they have no competing interests.

\section{Author details}

${ }^{1}$ Department of Global Health and Social Medicine, Harvard Medical School, Boston, USA. ${ }^{2}$ Socios En Salud Sucursal, Lima, Peru.

Received: 27 February 2019 Accepted: 13 June 2019

Published online: 20 June 2019

\section{References}

1. Wood RC, Luabeya AK, Weigel KM, Wilbur AK, Jones-Engel L, Hatherill M, et al. Detection of Mycobacterium tuberculosis DNA on the oral mucosa of tuberculosis patients. Sci Rep. 2015;5:8668.

2. Luabeya AK, Wood RC, Shenje J, Filander E, Ontong C, Mabwe S, et al. Non-invasive detection of tuberculosis by oral swab analysis. J Clin Microbiol. 2019:57(3):e01847-18.

3. Dorman SE, Schumacher SG, Alland D, Nabeta P, Armstrong DT, King B, et al. Xpert MTB/RIF Ultra for detection of Mycobacterium tuberculosis and rifampicin resistance: a prospective multicentre diagnostic accuracy study. Lancet Infect Dis. 2018;18(1):76-84.

4. Bisognin F, Lombardi G, Lombardo D, Carla Re M, Dal Monte P. Improvement of Mycobacterium tuberculosis detection by Xpert MTB/RIF Ultra: a head-to-head comparison on Xpert-negative samples. PLoS ONE. 2018;13(8):e020193.

5. Denkinger CM, Dolinger D, Schito M, Wells W, Cobelens F, Pai M, et al. Target product profile of a molecular drug-susceptibility test for use in microscopy centers. J Infect Dis. 2015;211(Suppl 2):S39-49.

\section{Publisher's Note}

Springer Nature remains neutral with regard to jurisdictional claims in published maps and institutional affiliations.

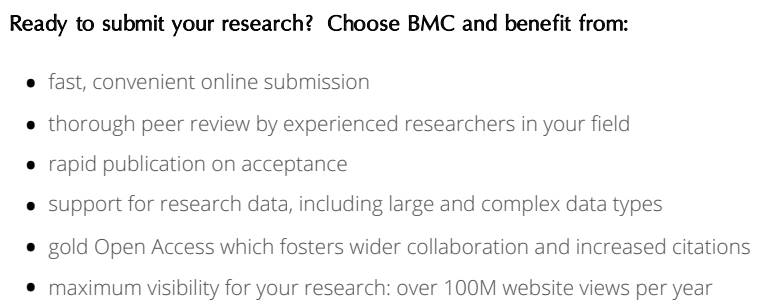

At $\mathrm{BMC}$, research is always in progress.

Learn more biomedcentral.com/submissions 